\title{
Long-Term Follow-Up after Surgical Management for Atypical Endometriosis: A Series of Nine Cases
}

\author{
Yasuhito Tanase $^{\mathrm{a}}$ Ryuji Kawaguchi ${ }^{\mathrm{a}}$ Tomoko Uchiyama ${ }^{\mathrm{b}}$ \\ Hiroshi Kobayashi ${ }^{a}$ \\ aDepartment of obstetrics and gynecology, Nara Medical University, Nara, Japan; \\ ${ }^{b}$ Department of diagnostic pathology, Nara Medical University, Nara, Japan
}

\section{Keywords}

Atypical endometriosis · Endometriosis associated ovarian cancer · Atypia

\begin{abstract}
Background and Objective: Atypical endometriosis is reported to possess a precancerous potential attributed to premalignant changes characterized by cytological atypia and architecture proliferation. Although the coexistence of atypical endometriosis and neoplasms has been reported, cases of atypical endometriosis transformation to carcinoma are rarely reported. The purpose of this case series was to evaluate the prognosis of atypical endometriosis. Subjects and Methods: Data from nine women who underwent surgical treatment including cystectomy and salpingo-oophorectomy with or without hysterectomy and diagnosed with atypical endometriosis was analyzed. Between January 2006 and January 2018, the clinical characteristics and prognosis of atypical endometriosis were evaluated. Results: During the follow-up period, eight of nine patients with atypical endometriosis did not develop malignant epithelial tumors, although one patient developed endometrioid carcinoma, grade 1, 48 months after her right
\end{abstract}




\section{Case Reports in Oncology}

Case Rep Oncol 2019;12:76-83

DOI: 10.1159/000496178

Tanase et al.: Long-Term Follow-Up after Surgical Management for Atypical

Endometriosis: A Series of Nine Cases

laparoscopic cystectomy. The median overall survival period for all patients was 68 (range 13131) months. Conclusion: When we encounter the cases of atypical endometriosis, it is necessary to consider the possibility of ovarian cancer and carefully follow those cases for long periods.

(C) 2019 The Author(s)

Published by S. Karger AG, Base

\section{Introduction}

Atypia of epithelium with endometriosis is often reported; it was first reported by Czernobilsky in 1978 [1], and LaGrenade and Silverberg proposed criteria for diagnosing atypical endometriosis (AE) in 1988 [2]. AE is diagnosed on the basis of histopathological criteria, including the features of eosinophilic cytoplasm, large hyperchromatic or pale nuclei with moderate to marked pleomorphism, an increased nuclear to cytoplasmic ratio, cellular crowding, and stratification or tufting. Many researchers have described AE as possessing a precancerous potential attributed to premalignant changes characterized by cytological atypia and architecture proliferation [3-5]. Moreover, the coexistence of AE and neoplasms has been often reported [6]. However, cases diagnosed as AE without neoplasms are rare, and there is no clinical guideline associated with them. Therefore, the management of such cases is often difficult. We identified nine consecutive cases of $\mathrm{AE}$ without neoplasms and followed them for long-term periods. Thus, the aim of this case series was to collect the clinical data and evaluate the long-term prognosis for $\mathrm{AE}$.

\section{Subjects and Methods}

Between January 2006 and January 2018, we studied nine women who underwent surgery and were diagnosed with AE without neoplasms at the Departments of Obstetrics and Gynecology, Nara Medical University, Japan, and its affiliated hospitals. Patients data included age at diagnosis, body mass index (BMI, $\left.\mathrm{kg} / \mathrm{m}^{2}\right)$, nulliparity, menopausal status, preoperative CA125 $(\mathrm{U} / \mathrm{mL})$ values, cyst size $(\mathrm{cm})$, laterality, the imaging characteristics of preoperative diagnosis, surgical procedure, and hormonal therapy after surgery. The follow-up periods (months) were evaluated, and recurrence and malignant transformation were analyzed during the periods. A recurrence of endometriosis was defined as the time when endometriomas over $2 \mathrm{~cm}$ in size were confirmed by MR imaging. A relapse of AE was defined as the situation in which the pathological finding of AE was confirmed. The follow-up periods were calculated from the date of surgical treatment to the time of the last follow-up visit.

$\mathrm{AE}$ was diagnosed on the basis of the following criteria: (i) the features of eosinophilic cytoplasm, (ii) large hyperchromatic or pale nuclei with moderate to marked pleomorphism, (iii) an increased nuclear to cytoplasmic ratio, (iv) cellular crowding, and (v) stratification or tufting. All pathological sections were analyzed by highly qualified pathologists with over 10 years of experience. We excluded cases diagnosed as borderline malignant ovarian tumors and ovarian carcinoma. The case report was approved by the ethics committee of Nara Medical University and written informed consent was obtained from all patients. 


\section{Case Reports in Oncology}

Case Rep Oncol 2019;12:76-83
\begin{tabular}{l|l}
\hline DOI: 10.1159/000496178 & $\begin{array}{l}\text { (c) 2019 The Author(s). Published by S. Karger AG, Basel } \\
\text { www.karger.com/cro }\end{array}$
\end{tabular}

Tanase et al.: Long-Term Follow-Up after Surgical Management for Atypical

Endometriosis: A Series of Nine Cases

\section{Results}

The clinicopathological features of the nine patients, including age, BMI, parity, menopausal status, and CA125 values are shown in Table 1 . The median age of patients at surgery was 39 (range 28-68) years, and eight (88.9\%) women were premenopausal. The median BMI was 20.5 (range 18.2-25.9) kg/m², and six (66.7\%) women were nulliparous. The preoperative CA125 level was elevated in four cases, and the median level was 30 (range 21-206) U/L.

The median follow-up periods for all patients were 68 (range 13-131) months. During the follow-up period, only one patient experienced a recurrence of endometriosis. The recurrence occurred 7 months after the patient underwent surgery. Finally, the patient developed stage 1C endometrioid carcinoma, grade 1, 48 months after undergoing right laparoscopic cystectomy. We reported the case in 2013 [7]. There were no case of recurrence of AE.

Preoperative diagnosis was performed by pelvic Magnetic Resonance Imaging (MRI) in seven patients and by computed tomography in one patient. The median cyst size was $6.8 \mathrm{~cm}$ (range 3.5-9.2) and only one patient revealed a multi lobular cyst. Almost all the cysts showed the same signal pattern with regard to the MRI intensity; T1-weighted images had high signal intensity and T2-weighted images had iso-high signal intensity. The signal patterns indicated the so-called chocolate cyst. Malignancy was suspected preoperatively in only one patient (case 2) because her cyst had a small mural nodule that was enhanced with gadrium (Fig. 1). Except for this one case, we could not identify mural nodules in the cysts of other patients, which would have indicated a possible malignant transformation of ovarian endometriosis.

The curative surgery for endometriosis, hysterectomy, and bilateral salpingo-oophorectomy was performed in only one case, and the conservative surgery, one-sided or bilateral cystectomy, or one-sided salpingo-oophorectomy with or without hysterectomy was performed in the other eight cases. The patient in whom a recurrence and malignant transformation was identified underwent the conservative surgery (Table 2).

With regard to laterality of $\mathrm{AE}$, six of the nine cases $(66.7 \%)$ were left-sided and three were right-sided (Table 1). Postoperative hormonal therapy was administered to control recurrence in five of the eight cases who received conservative therapy; one case received gonadotropin-releasing hormonal agonist (GnRHa), two received dienogest and two others received low dose estrogen-progestin. The case whose condition developed to ovarian endometrioid carcinoma was administered GnRHa therapy. Neither of them received adjuvant chemotherapy nor radical surgery including retroperitoneal lymphadenectomy after their surgeries (Table 2).

Histology of the nine cases is shown in Figure 2. All the nine patients fulfilled the criteria of AE. All the patients are still alive, and the survival rate is $100 \%$.

\section{Discussion}

A subset of $\mathrm{AE}$ has been considered to coexist with ovarian carcinoma, especially endometriosis-associated ovarian carcinoma, including clear-cell carcinoma and endometrioid carcinoma with a potential of developing or transforming to ovarian carcinoma [8]. The transformation rate into ovarian cancer, however, remains unclear. There have been no reports regarding the prognosis of $\mathrm{AE}$ without neoplasms. Hence, the present report attempted to 


\section{Case Reports in Oncology}

Case Rep Oncol 2019;12:76-83

DOI: $10.1159 / 000496178$

(C) 2019 The Author(s). Published by S. Karger AG, Base www.karger.com/cro

Tanase et al.: Long-Term Follow-Up after Surgical Management for Atypical

Endometriosis: A Series of Nine Cases

investigate the prognosis of $\mathrm{AE}$ without neoplasms for long-term follow-up periods by evaluating the incidences of malignant transformation and recurrence of endometriosis or AE. In our present report, only one of the nine patients (11.1\%) experienced a recurrence of endometriosis, and developed stage $1 \mathrm{C}$ endometrioid carcinoma.

There are two important issues concerning AE cases. One is that we need to consider the possibility of its coexistence with neoplasms, and the other is determining how to conduct a follow-up of those patients. Therefore, strict differential diagnosis is needed. There are neither clinical guidelines regarding postoperative monitoring, such as counseling for hormonal therapy (e.g., oral contraceptives and luteal hormonal therapy) nor rules of curative surgery including total hysterectomy and bilateral salpingo-oophorectomy following conservative primary surgery. In addition, there is no scientific evidence indicating that chemotherapy is effective or whether radical surgery, including retroperitoneum lymphadenectomy, is necessary for the treatment of AE. Prudent and long-term follow-up is needed.

The incidence of $\mathrm{AE}$ without neoplasm was reported to be 3.6\% (7/194) by Czernobilsky and Morris [1], 1.7\% (4/255) by Fukunaga et al. [3], 32.3\% (34/105) by Seidmann [9], and $5.8 \%(7 / 120)$ by Bayramoglu and Duzcan [10]. In contrast, the incidence of AE with neoplasm was reported to be $22.8 \%$ (29/127) by Ogawa et al. [11], 14.7\% (33/224) by Fukunaga et al. [3], and 4.4\% (8/183) by Oral et al. [6]. These studies agreed that the so-called atypical endometriosis may represent a step in the carcinogenic pathway. However, factors leading to atypia remain poorly understood.

There are few reports of $\mathrm{AE}$ without neoplasm, but some researchers reported cases of $\mathrm{AE}$ that had developed to ovarian carcinoma. Moll et al. reported the first case of atypical endometriosis that underwent complete malignant transformation within 3 years and subsequently gave rise to invasive clear-cell carcinoma [4]. In a follow-up study, Fukunaga et al. described one of four patients with AE who developed endometrioid carcinoma in the abdominal wall 18 months after they underwent left oophorectomy [3]. Furthermore, Leng et al. reported a case of carcinosarcoma arising from $\mathrm{AE}$ that developed within 16 years because of cesarean scarring [5]. We reported a case whose condition had progressed from an endometriotic cyst with atypical feature to endometrioid carcinoma within 10 years [7]. As followed in cases, it is important to conduct long-term follow-up of AE without neoplasms.

There were no characteristic findings with regard to preoperative diagnoses. AE did not always show mural nodules in the inner wall of cysts, which are typical findings that indicate a possible malignant transformation of endometriosis. One of the nine cases showed multilobular cysts, and another one case presented a very small mural nodule on the cyst, which was enhanced by gadium (Fig. 1).

The incidence of malignant transformation of typical endometriosis has been reported to be approximately $1 \%$ [12]. Considering these reports and the low incidence of AE, we could assume that the incidence of malignant transformation of $\mathrm{AE}$ is higher than that of typical endometriosis. Therefore, it is essential to manage cases diagnosed as AE with care, especially in younger age, as they need to be followed up for long-term periods by considering precancerous characteristics.

Low dose estrogen-progestin (LEP) has been reported to reduce the occurrence of ovarian cancer [13], and hormonal therapy including LEP and luteal hormonal therapy for benign endometriotic tissue is effective to suppress the recurrence of endometriosis [14]. On the basis of these results, hormonal therapy for AE, LEP, and luteal hormonal therapy including 
dienogest, could also have the same effect of reducing the incidence of AE recurrence and malignant transformation.

The case whose condition had developed to ovarian endometrioid carcinoma, had received hormonal therapy with GnRHa for several months after the surgeries. Over 10 years ago, GnRHa was the mainstream hormonal therapy for endometriosis in our country, and LEP or luteal hormonal therapy was not popular. Hormonal therapy including LEP and luteal hormonal therapy for $\mathrm{AE}$ might be recommended for the purpose of reducing the recurrence in the same way as used in treating typical endometriosis; GnRHa is not recommended because of its inability to be used for long-term periods and the lack of evidence with regard to its effectiveness. Patients who wish for a baby are encouraged to prioritize pregnancy. We do not recommend postoperative chemotherapy because of lack of evidence supporting its effectiveness. In the present study, patients did not receive postoperative chemotherapy.

The recurrence of endometriosis occurs at a high frequency; it is estimated that the frequency after surgery increases by approximately $10 \%$ every year. There have been, however, few reports on the frequency of AE recurrences. In the present study only one case exhibited a recurrence of endometriosis 6 months after her surgery which finally developed to ovarian cancer. All the cases, however, are still alive; the survival rate was $100 \%$.

LaGrenade and Silverberg defined the criteria for $\mathrm{AE}$, but it has not become popular and is still controversial. Atypia with endometriosis is sometimes confirmed pathologically, and the degree of atypia is different in each case. Basically, chronic inflammatory changes occur in endometriotic tissues and are often confirmed by metaplastic reaction in the epithelium of ovarian benign endometrioma. Those changes are diagnosed as part of a benign component, and are often neglected clinically. It sometimes becomes problematic to differentiate AE from metaplastic changes, but this could be distinguished on the basis of the degree of atypia. Perhaps, the metaplastic change in epithelium of endometriosis is a pre-change and pre-state of $\mathrm{AE}$.

Although the pathogenesis of malignant changes in endometriosis remains unclear, some studies suggest that certain aspects of endometriosis are similar to those of other malignancies $[8,15]$. There are numerous reported cases of malignancies arising from endometriotic deposits and substantial histological evidence confirming that endometriosis is associated with ovarian cancer, particularly endometrioid carcinoma and clear-cell carcinoma. Reportedly, AE possesses a precancerous potential and numerous studies have reported malignant transformations in endometriosis. Moreover, the coexistence of endometriosis and neoplasms has been reported. However, cases of AE transforming to carcinoma are rarely reported.

\section{Acknowledgment}

The authors thank S. Sugimoto (Nara Medical University, Nara) for her assistance with the collection of the data of pathology.

\section{Statement of Ethics}

The authors have no ethical conflicts to disclose. 


\section{Disclosure Statement}

The authors declared no potential conflicts of interest with respect to the research, authorship, and/or publication of this article.

\section{Funding Sources}

The authors received no financial support for the research, authorship, and/or publication of this article.

\section{Author Contributions}

Data collection and drafting of the manuscript, YT; Important intellectual content and statistical analysis, RK; Diagnosis and analysis of pathology TU; Original proposer and proof, HK; All authors approved final version of manuscript.

\section{References}

1 Czernobilsky B, Morris WJ. A histologic study of ovarian endometriosis with emphasis on hyperplastic and atypical changes. Obstet Gynecol. 1979 Mar;53(3):318-23.

2 LaGrenade A, Silverberg SG. Ovarian tumors associated with atypical endometriosis. Hum Pathol. 1988 Sep;19(9):1080-4.

3 Fukunaga M, Nomura K, Ishikawa E, Ushigome S. Ovarian atypical endometriosis: its close association with malignant epithelial tumours. Histopathology. 1997 Mar;30(3):249-55.

4 Moll UM, Chumas JC, Chalas E, Mann WJ. Ovarian carcinoma arising in atypical endometriosis. Obstet Gynecol. 1990 Mar;75(3 Pt 2):537-9.

5 Leng J, Lang J, Guo L, Li H, Liu Z. Carcinosarcoma arising from atypical endometriosis in a cesarean section scar. Int J Gynecol Cancer. 2006 Jan-Feb;16(1):432-5.

6 Oral E, Ilvan S, Tustas E, Korbeyli B, Bese T, Demirkiran F, et al. Prevalence of endometriosis in malignant epithelial ovary tumours. Eur J Obstet Gynecol Reprod Biol. 2003 Jul;109(1):97-101.

7 Tanase Y, Furukawa N, Kobayashi H, Matsumoto T. Malignant Transformation from Endometriosis to Atypical Endometriosis and Finally to Endometrioid Adenocarcinoma within 10 Years. Case Rep Oncol. 2013 Sep;6(3):480-4.

8 Krawczyk N, Banys-Paluchowski M, Schmidt D, Ulrich U, Fehm T. Endometriosis-associated Malignancy. Geburtshilfe Frauenheilkd. 2016 Feb;76(2):176-81.

9 Seidman JD. Prognostic importance of hyperplasia and atypia in endometriosis. Int J Gynecol Pathol. 1996 Jan;15(1):1-9.

10 Bayramoğlu H, Düzcan E. Atypical epithelial changes and mutant p53 gene expression in ovarian endometriosis. Pathol Oncol Res. 2001;7(1):33-8.

11 Ogawa S, Kaku T, Amada S, Kobayashi H, Hirakawa T, Ariyoshi K, et al. Ovarian endometriosis associated with ovarian carcinoma: a clinicopathological and immunohistochemical study. Gynecol Oncol. 2000 May;77(2):298-304.

12 Kobayashi H, Sumimoto K, Kitanaka T, Yamada Y, Sado T, Sakata M, et al. Ovarian endometrioma -risks factors of ovarian cancer development. Eur J Obstet Gynecol Reprod Biol. 2008 Jun;138(2):187-93.

13 Shafrir AL, Schock H, Poole EM, Terry KL, Tamimi RM, Hankinson SE, et al. A prospective cohort study of oral contraceptive use and ovarian cancer among women in the United States born from 1947 to 1964. Cancer Causes Control. 2017 May;28(5):371-83.

14 Vercellini P, Buggio L, Berlanda N, Barbara G, Somigliana E, Bosari S. Estrogen-progestins and progestins for the management of endometriosis. Fertil Steril. 2016 Dec;106(7):1552-1571.e2. 


\section{Case Reports in Oncology}

\begin{tabular}{l|l} 
Case Rep Oncol 2019;12:76-83 \\
\hline DOI: 10.1159/000496178 & $\begin{array}{l}\text { (C) 2019 The Author(s). Published by S. Karger AG, Basel } \\
\text { www.karger.com/cro }\end{array}$
\end{tabular}

Tanase et al.: Long-Term Follow-Up after Surgical Management for Atypical Endometriosis: A Series of Nine Cases

15 Reid BM, Permuth JB, Sellers TA. Epidemiology of ovarian cancer: a review. Cancer Biol Med. 2017

Feb;14(1):9-32.
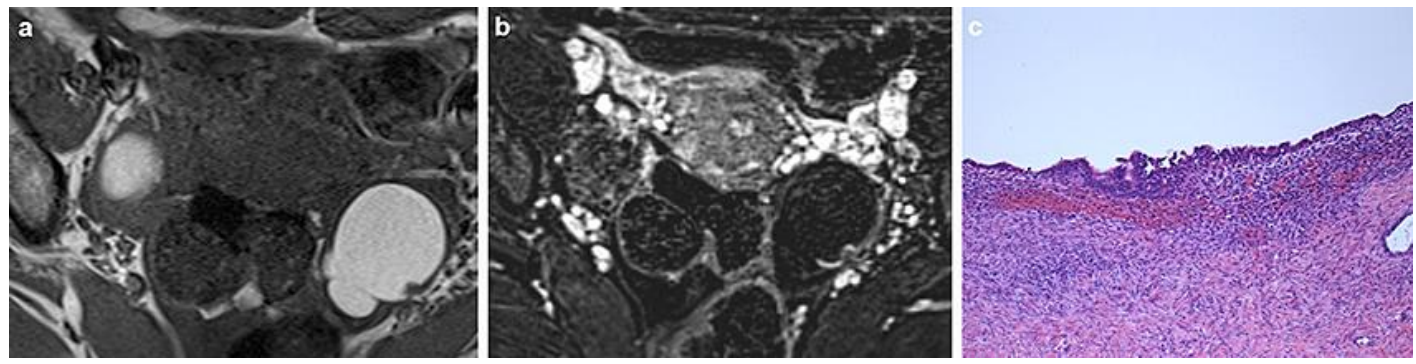

Fig. 1. a On axial T1-weighted images of the case 2, left sided mass demonstrates high signal intensity with mural nodule on the left side wall of the cyst. $\mathbf{b}$ The mural nodules was enhanced on gadolinium enhanced fat-suppressed T1-weighted image. c Epithelial cells with hyperchromatic and increased nuclear to cytoplasmic ratio showed stratification and tufting on the cyst wall.
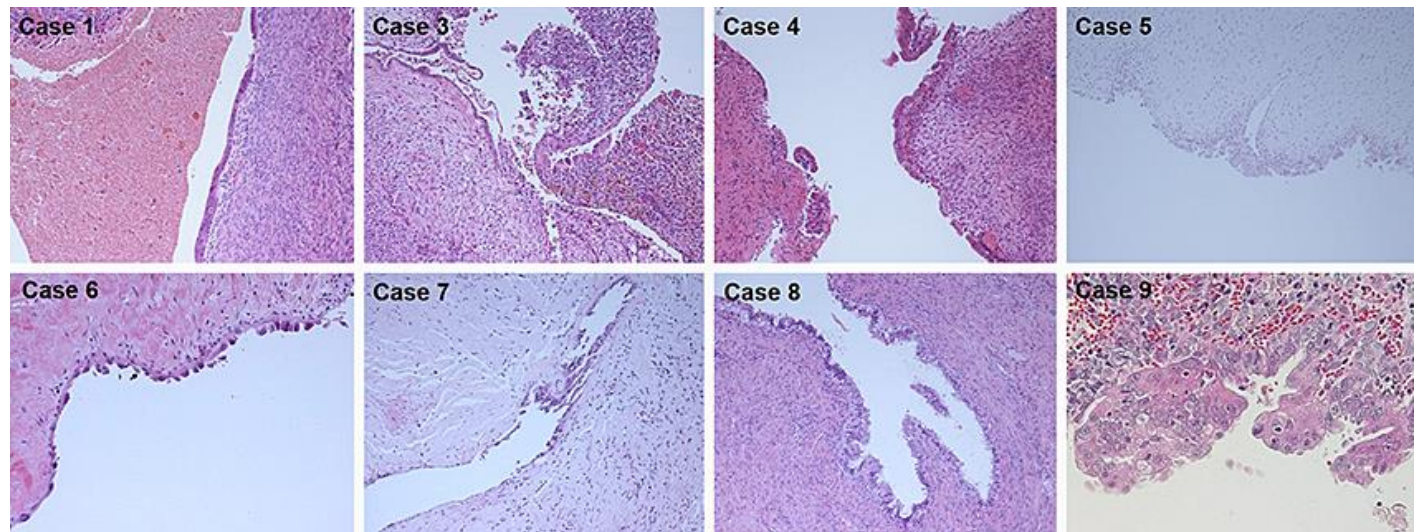

Fig. 2. Histology of all cases without case 2. HE. All section had some kind of atypical features. AE is diagnosed on the basis of histopathological criteria, including the features of eosinophilic cytoplasm, large hyperchromatic or pale nuclei with moderate to marked pleomorphism, an increased nuclear to cytoplasmic ratio, cellular crowding, and stratification or tufting. 


\section{Case Reports in Oncology}

anase et al: Long-Term Follow-Up after Surgical Management for Atypical

Endometriosis: A Series of Nine Cases

Table 1. Characteristics of the patients

\begin{tabular}{|c|c|c|c|c|c|c|c|c|}
\hline Case & $\begin{array}{l}\text { Age, } \\
\text { years }\end{array}$ & Parity & Menopausal status & $\begin{array}{l}\mathrm{BMI}, \\
\mathrm{kg} / \mathrm{m}^{2}\end{array}$ & Laterality & $\begin{array}{l}\text { Cyst size, } \\
\mathrm{cm}\end{array}$ & $\begin{array}{l}\text { CA125, } \\
\text { IU/L }\end{array}$ & $\begin{array}{l}\text { Follow-up } \\
\text { periods, } \\
\text { months }\end{array}$ \\
\hline 1 & 46 & 0 & Premenopausal & 18.9 & Right & 4.1 & 48 & 64 \\
\hline 2 & 39 & 3 & Premenopausal & 24.4 & Left & 3.5 & 131 & 60 \\
\hline 3 & 43 & 0 & Premenopausal & 18.6 & Left & 7.0 & 22 & 68 \\
\hline 4 & 31 & 0 & Premenopausal & 19.7 & Left & 8.6 & 25 & 57 \\
\hline 5 & 42 & 1 & Premenopausal & 18.2 & Left & 8.1 & 21 & 92 \\
\hline 6 & 28 & 0 & Premenopausal & 20.7 & Left & 9.3 & 35 & 103 \\
\hline 7 & 36 & 0 & Premenopausal & 20.5 & Right & 8.2 & 206 & 88 \\
\hline 8 & 68 & 2 & Postmenopausal & 25.9 & Left & 20 & 27 & 13 \\
\hline 9 & 29 & 0 & Premenopausal & 20.5 & Right & 5 & 30 & 131 \\
\hline
\end{tabular}

Table 2. Preoperative diagnosis, treatment and outcome of the patients

\begin{tabular}{llllll}
\hline Case & $\begin{array}{l}\text { Surgical } \\
\text { procedure }\end{array}$ & Hormonal therapy & $\begin{array}{l}\text { Recurrence of } \\
\text { endometriosis }\end{array}$ & $\begin{array}{l}\text { Recurrence } \\
\text { of AE }\end{array}$ & $\begin{array}{l}\text { Malignant } \\
\text { transformation }\end{array}$ \\
\hline 1 & Conservative & None & None & None & None \\
2 & Conservative & LEPa (4 months) & None & None & None \\
3 & Conservative & Dienogest (2 years) & None & None & None \\
4 & Conservative & None & None & None & None \\
5 & Conservative & Dienogest (3 years) & None & None & None \\
6 & Conservative & LEPa (3 years) & None & None & None \\
7 & Conservative & None & None & None & None \\
8 & Curative & None & None & None & None \\
9 & Conservative & GnRHa & + & None & + \\
& & $(6$ months $) \times 3$ course & & & \\
\hline
\end{tabular}

${ }^{a}$ Low dose estrogen-progestin, $\mathrm{b}$ Gonadotropin-releasing hormonal agonist. Curative surgery includes hysterectomy and bilateral salpingo-oophorectomy. Conservative surgery includes one-sided or bilateral cystectomy or one-sided salpingo-oophorectomy with or without hysterectomy. 